\title{
Análisis de la Tensión Interfacial Entre Dos Fluidos Inmiscibles con el Uso de Cuatro Surfactantes (Catiónico, No-Iónico), de la Arena Productora U Inferior del Campo Pindo
}

\author{
Celi, Jonathan 1, * iD ; Gómez, Franklin ${ }^{1}$ iD ; Enríquez, Bolívar ${ }^{2}$ iD

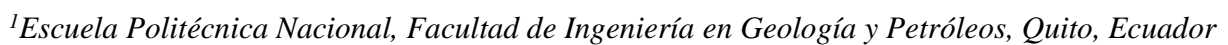 \\ ${ }^{2}$ Universidad Central del Ecuador, Facultad en Geología, Minas Petróleos y Ambiental, Quito, Ecuador
}

\begin{abstract}
Resumen: La aplicación de recuperación mejorada puede incrementar el factor de recobro en más de un $20 \%$ después de aplicar recuperación primaria y secundaria. Para recuperar ese petróleo remanente de los reservorios se puede inyectar fluidos con surfactantes, lo cual está dentro de la recuperación terciaria. En este trabajo, se examina las tensiones interfaciales y superficiales con el uso de cuatro surfactantes comerciales en diferentes concentraciones. Generando curvas para analizar la tensión interfacial entre dos fluidos inmiscibles (agua de inyección con surfactante y petróleo). La investigación se desarrolló en tres fases. En la primera, se analizaron las propiedades del agua (salinidad y concentración de sólidos disueltos), surfactante ( $\mathrm{pH}$ y el análisis de espectrometría infrarroja) y petróleo (BSW y API). En la segunda fase se midieron las tensiones superficiales (TS) en una mezcla de agua de formación y surfactante a diferentes concentraciones para obtener la concentración micelar crítica (CMC), y en la tercera fase la medición de tensiones interfaciales (IFT) en los dos fluidos inmiscibles. Es decir, una mezcla de agua de inyección, surfactante y petróleo del campo Pindo. Las muestras de fluidos fueron proporcionadas por Petrosud Petroriva. Con los resultados, se procedió a generar curvas de Tensión superficial e interfacial Vs Concentración. El surfactante HALMX1528052 de tipo no iónico con grupos funcionales de Alquil e Hidróxilo a una concentración del $0.3 \%$ en peso, tuvo la mayor reducción tanto en la CMC e IFT.
\end{abstract}

Palabras claves: Concentracion Micelar Crítica, Espectometría Infrarroja, Inmiscibles, Surfactante, Tensión Interfacial

\section{Analysis of the Interfacial Tension Between Two Immiscible Fluids with the Use of Four Surfactants (Cationic, Non-Ionic), From the "Ui" Producing Sand of the Pindo Field}

\begin{abstract}
The application of enhanced oil recovery can increase the recovery factor by more than $20 \%$, after the use of primary and secondary recovery. For the recuperation of this remnant oil inside the reservoirs, it can inject fluids with a surfactant, which is inside of enhanced oil recovery. This research was conducted to examine the interfacial and superficial tensions with the use of four commercial surfactants in different concentrations. Generating curves to analyze the variation of interfacial tension between two immiscible fluids (injection water with surfactant and petroleum). This work was developed in three phases. In the first one, were analyzed the properties of water (salinity and concentration of dissolved solids), surfactant ( $\mathrm{pH}$ and infrared spectrometry), and petroleum (API). In the second phase, the superficial tension (ST) was measured in a mixture of injection water and surfactant at different concentrations in order to obtain their critical micellar concentration (CMC). Finally, in the third phase, the measurement of interfacial tensions (IFT) in a mixture of the immiscible fluids. That is a mixture of injection water, surfactant, and oil from the Pindo field. Fluid samples were provided by Petrosud Petroriva company. With the results, it was proceeded to generate curves of superficial and interfacial tension Vs concentration. The surfactant HALMX152805-2, which is a nonionic type with functional groups of alkyl and hydroxyl with $0,3 \%$ of weight concentration, had the greatest reduction in both CMC and IFT.
\end{abstract}

Keywords: Immiscible, Infrared Spectroscopy, Interfacial Tension, Micelar Critical Concentration, Surfactant.

\section{INTRODUCCIÓN}

En Ecuador se han buscado soluciones para cubrir la demanda diaria de petróleo por falta de nuevos pozos o por el declive de la producción de los reservorios depletados (Morales, 2010). La extracción de petróleo de un yacimiento se realiza en tres etapas de recuperación. Con la recuperación primaria se llega a extraer hasta un $24 \%$ de petróleo de un reservorio (Bolívar, et al., 2017). La recuperación secundaria mediante la inyección de agua o gas, permite aumentar el factor de recobro hasta un $15 \%$ adicional al de la recuperación primaria (Bolívar, et al., 2017). Finalmente, se pueden realizar trabajos de

*jonathanceli@hotmail.com

Recibido: 06/11/2019

Aceptado: 23/03/2021

Publicado: 31/05/2021

$10.33333 /$ rp.vol47n2.03

CC BY 4.0 
recuperación terciaria, utilizando métodos como la inyección de químicos o trabajos térmicos. De esta manera se puede recuperar hasta un $20 \%$ adicional del hidrocarburo, reduciendo la viscosidad del fluido y mejorando el flujo dentro del yacimiento (Abubaker, et al., 2015).

La investigación se centra en la recuperación terciaria mediante el uso de surfactantes. Y se analizaron las tensiones interfaciales en una mezcla de petróleo y agua de inyección con surfactantes.

Los surfactantes son sustancias químicamente compuestas de moléculas anfifílicas con una parte polar positiva o negativa y la otra no polar (Salager, et al., 2007). El uso de surfactantes ha tenido éxito por su menor costo en comparación a la recuperación térmica, ya que al usar bajas concentraciones en rangos de $0,1 \%$ a $0,3 \%$ en peso se puede reducir la adsorción del surfactante adicionado y evitar la creación de emulsiones. El objetivo de los surfactantes es disminuir la acción de las fuerzas intermoleculares y aumentar la movilidad de los fluidos dentro del yacimiento (Chuck y Trombetta, 2007). Para inyectar estos químicos dentro del pozo es necesario analizar qué tipo de surfactante se utilizará, puesto que existen cuatro clases, que son: aniónicos, catiónicos, no iónicos y anfotéricos (Salager, 2002). Cada surfactante tiene diferentes tipos de grupos funcionales y para identificarlos es necesario realizar un análisis de espectro infrarrojo (IR). El IR consiste en la interacción entre la radiación infrarroja y las moléculas existentes dentro del fluido, determinando así las longitudes de onda que tiene cada molécula (Macho, 2002).

Inicialmente se analizaron propiedades como: los sólidos disueltos, la salinidad del agua de inyección y el porcentaje de agua y sedimentos BSW (por sus siglas en Inglés Basic Sedimental Water) contenidos en el hidrocarburo. Posteriormente, se realizaron ensayos de tensión superficial (TS) en una mezcla de agua de inyección con surfactante, con el objetivo de determinar la concentración micelar crítica (CMC), que es la mínima cantidad de surfactante para la formación de micelas (Novelo y Fadrique, 2005), y la CMC fue corroborada con la medición de la conductividad. A continuación, se llevó a cabo pruebas de Tensión Interfacial (IFT) con un tensiómetro de anillo Du Noüy, que mide la fuerza que ejerce entre las fases líquido - líquido o gas líquido (Salager, 2005).

Finalmente, con las mediciones obtenidas se generaron diferentes curvas, mostrando así, que el surfactante comercial HALMX152805-2 (no iónico del grupo alquil) presenta una mayor reducción en la tensión interfacial. Los ensayos de laboratorio se realizaron a condiciones de presión de $0,708 \mathrm{~atm}$ y una temperatura de $68{ }^{\circ} \mathrm{F}$. Para condiciones de yacimiento donde presiones y temperaturas pueden llegar a niveles extremos, los valores de la CMC van a variar debido a que la solubilidad tiende a aumentar y por consiguiente la IFT (Salager,1993).

\section{MARCO TEÓRICO}

En la provincia de Orellana a una distancia de $150 \mathrm{Km}$ de Lago Agrio y a $10 \mathrm{Km}$ al oeste del campo Auca se encuentra ubicado el Campo Pindo, con un área de 6800 hectáreas (Villegas, 2013).

Los campos Pindo y Palanda Yuca Sur tienen un área aproximada de $64 \mathrm{Km}^{2}$ y $140 \mathrm{Km}^{2}$ estructuradas en: Pindo, Pindo Este, Yuca Sur, Palanda y Primavera (Rodríguez, 2015). Las arenas productoras del campo son Basal Tena, "U" superior e inferior, " $\mathrm{T}$ ” inferior y Hollín superior e inferior.

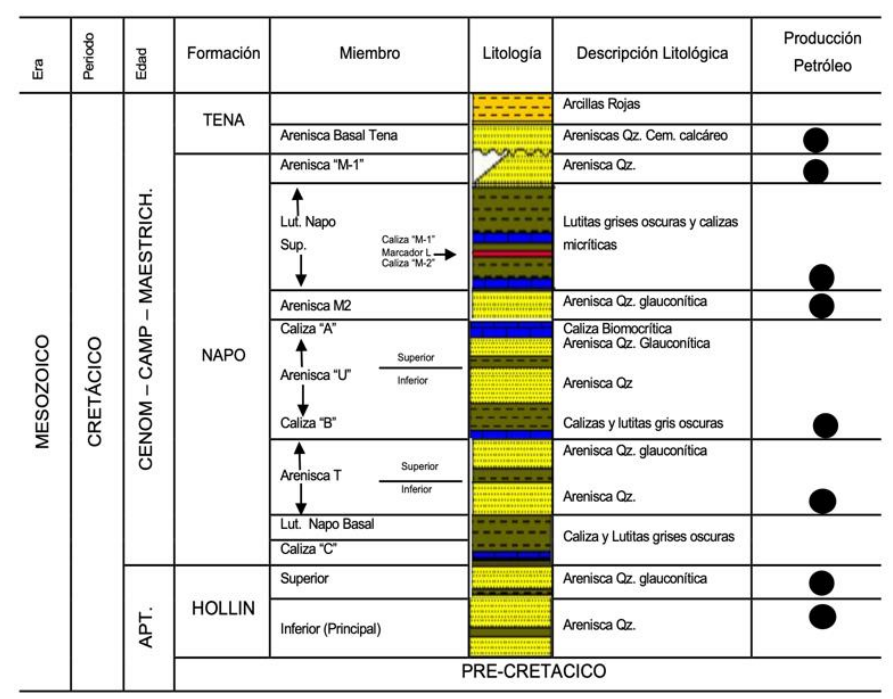

Figura 1. Mapa estructural Campo Pindo (Fuente: Consorcio Petrosud Petroriva, 2010)

Actualmente, el campo Pindo posee 17 pozos productores, 3 pozos reinyectores y 1 pozo inyector, con un factor de recobro del $28 \%$, y un \%BSW promedio del $0.51 \%$ (Consorcio Petrosud Petroriva, 2018-a). Las propiedades de sus fluidos están descritas en la Tabla 1.

Tabla 1. Propiedades de los pozos Pindo 11, 13 y 17. De la arena Ui (Fuente: Consorcio Petrosud - Petroriva, 2018-b)

\begin{tabular}{|c|c|c|c|c|c|}
\hline \multirow{12}{*}{ 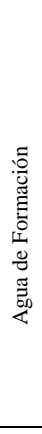 } & Pozo & & PIN 11 & PIN 13 & PIN-17 \\
\hline & $\mathrm{Na}^{+}$ & $(\mathrm{mg} / \mathrm{l})$ & 28500 & 27400 & 25175 \\
\hline & $\mathrm{Mg}^{2+}$ & $(\mathrm{mg} / \mathrm{l})$ & 510 & 340 & 534 \\
\hline & $\mathrm{Ca}^{2+}$ & $(\mathrm{mg} / \mathrm{l})$ & 4,800 & 2,800 & 3,920 \\
\hline & $\mathrm{Ba}^{2+}$ & $(\mathrm{mg} / \mathrm{l})$ & 40 & 20 & 8 \\
\hline & $\mathrm{Fe}$ & $(\mathrm{mg} / \mathrm{l})$ & 22 & 9 & 43 \\
\hline & $\mathrm{Cl}^{-}$ & $(\mathrm{mg} / \mathrm{l})$ & 53,833 & 48,000 & 47,000 \\
\hline & $\mathrm{SO}_{4}{ }^{2-}$ & $(\mathrm{mg} / \mathrm{l})$ & 10 & 50 & 86 \\
\hline & Bicarbonatos (Alcalinidad) & $(\mathrm{mg} / \mathrm{l} \mathrm{HCO} 3)$ & 232 & 306 & 450 \\
\hline & Ácidos carboxílicos & $(\mathrm{mg} / \mathrm{l})$ & 0.00 & 0.00 & 0.00 \\
\hline & TDS (Calculado) & $(\mathrm{mg} / \mathrm{l})$ & 88058 & 79055 & 77253 \\
\hline & pH STD & --- & 6.21 & 6.25 & 7.00 \\
\hline \multirow{8}{*}{ 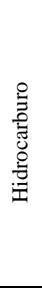 } & $\mathrm{CO}_{2} \mathrm{Gas}$ & $(\%)$ & 6 & 3 & 8 \\
\hline & $\mathrm{H}_{2} \mathrm{~S}$ Gas & $(\mathrm{ppm})$ & 7 & 10 & 7 \\
\hline & Gas/Día & (MSCFPD) & 47 & 46 & 49 \\
\hline & $\mathrm{CO}_{2}$ Agua & $(\mathrm{mg} / \mathrm{l})$ & 352 & 352 & 352 \\
\hline & $\mathrm{H}_{2} \mathrm{~S}$ Agua & $(\mathrm{mg} / \mathrm{l})$ & 0.1 & 0.1 & 0.1 \\
\hline & Agua/Día & (BWPD) & 1,862 & 276 & 608 \\
\hline & Oil/Día & (BOPD) & 328 & 784 & 342 \\
\hline & Densidad API & ${ }^{\circ} \mathrm{API}$ & 17.9 & 17.3 & 19.5 \\
\hline
\end{tabular}


En el campo Pindo se realizan trabajos de recuperación secundaria como la inyección de agua, pero las producciones de los pozos están en declive; razón por lo que se investiga el uso de surfactantes y polímeros para su implementación esperando tener mejores resultados en el factor de recobro (Consorcio Petrosud Petroriva, 2018). La producción promedio de los tres pozos se presenta en la Figura 2.

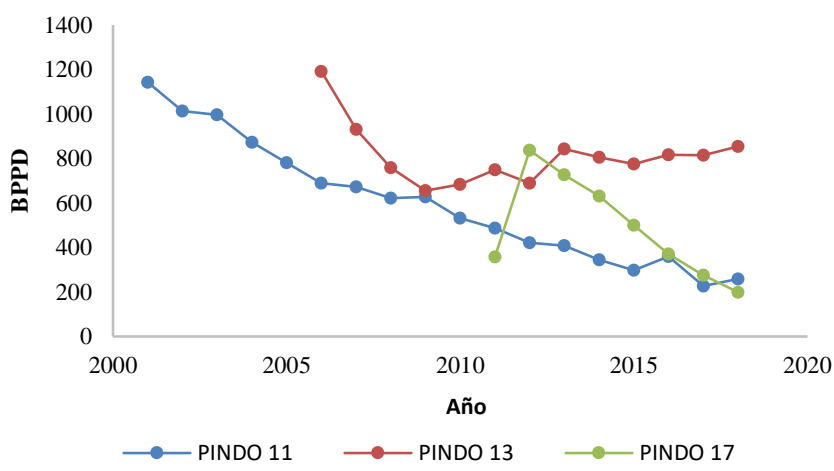

Figura 2. Promedio de producción de los pozos Pindo (Fuente: Consorcio Petrosud Petroriva, 2018-d)

\subsection{Tipos de recuperación}

Un yacimiento petrolero almacena: agua, petróleo y en ciertos casos gas, estos deben estar en un medio poroso de una roca almacén, la cual puede tener un origen sedimentario de diferentes tipos como son areniscas o calizas, consolidadas o no. Llegando a alcanzar hasta un $40 \%$ en su proporción volumétrica (Salager, 2005).

Luego de la perforación, con la recuperación primaria se puede extraer hasta un $24 \%$ de petróleo (Bolívar, et al., 2017); el pozo produce a flujo natural impulsado por la diferencia de presiones, los mecanismos naturales de producción existentes son: empuje por agua, empuje por gas disuelto y la expansión del casquete de gas. Estos mecanismos se mantienen durante un tiempo hasta ver un declive en su producción causado por pérdida de presión en el yacimiento. (Schlumberger, 2018 a).

Al momento que la presión del yacimiento declina y la energía natural no es suficiente para mantener la producción, se procede a recurrir a métodos de bombeo artificial, que consiste en bajar diferentes tipos de mecanismos como el bombeo mecánico, bombeo hidráulico, gas - lift y bombeo electro sumergible (Hernández, 2008).

Terminada la recuperación primaria se procede con la secundaria que sirve para restablecer o mantener las presiones dentro del reservorio (Almeida, et al., 2015); trabajos que consisten en inyectar agua a través de un pozo inyector, aumentando la energía del yacimiento con un mecanismo de desplazamiento generando un barrido del petróleo residual y su salida es por un pozo productor; con este método se puede llegar a extraer hasta un $15 \%$ adicional en las reservas existentes dentro del yacimiento (Hernández, 2008).

Cuando los trabajos de recuperación secundaria ya no son factibles, puede existir hasta un $65 \%$ de petróleo remanente entrampado en los poros del reservorio (SNF FLOERGER, 2016). Por esta razón, se analiza la recuperación mejorada, con propósitos, como: la restauración de la presión de la formación o mejorar el desplazamiento de fluidos dentro del yacimiento (Schlumberger, 2018 b).

La recuperación mejorada tiene dos tipos de trabajos: los métodos térmicos como la inyección cíclica o continua de vapor y la combustión in situ, y la inyección de agua con productos químicos como el método miscible (solventes, $\mathrm{CO}_{2}$, micro emulsiones), métodos de baja tensión (surfactantes), métodos alcalinos, inyección de agua viscosa (polímeros) y combinación de los tres anteriores (ASP) (Salager, 2005). Para la aplicación de cualquier método se debe realizar un estudio exhaustivo y considerar variables como el buzamiento, la profundidad, la continuidad y la cantidad de gas existente (Rondón, 2019). Otros factores que también se consideran son: temperatura, presión, permeabilidad $(\mathrm{K})$, porosidad $(\varnothing)$, saturaciones de agua ( $\mathrm{Sw}$ ) y de petróleo (So), pH viscosidad y gravedad API. Estas propiedades son importantes para conocer el tipo de operación a realizar en el yacimiento (Schlumberger, 2018b).

\subsection{Surfactantes}

Los surfactantes son sustancias químicamente compuestas de moléculas anfifílicas; es decir, moléculas de dos polaridades, una parte polar y la otra no polar o apolar (Salager, 2007). Cada una de estas partes actúa de diferente manera, la parte polar está compuesta de uno o varios grupos funcionales polares como el sulfato, sulfonato, ácido, fosfato, amina, alcohol, etc., y la parte apolar es una cadena de hidrocarbonada lineal o ramificada de tipo alquil o alquil benceno que en ciertos casos puede contener átomos de halógeno u oxígeno (Salager, 2002), el núcleo depende de la composición química del surfactante. Como los surfactantes poseen doble afinidad las moléculas deben satisfacer sus afinidades, la parte polar o hidrofílica debe estar en contacto con un solvente polar como el agua y la parte apolar o hidrofóbica debe estar en un solvente orgánico como el petróleo. Estas condiciones solo se las encuentra en la frontera de dos fases como pueden ser una fase condensada y una gaseosa (superficie) o en una interfase; es decir en la frontera compuesta por dos líquidos o un líquido y un sólido (Salager y Fernández, 2004).

El efecto de los surfactantes es la reducción de la tensión interfacial y superficial; actuando como un detergente ya que al momento que se encuentra en la interfase o superficie disminuye la fuerza de atracción entre las moléculas que existen en un líquido sin desequilibrar el sistema (Antón, 2005).

Los surfactantes se clasifican de acuerdo a la estructura de su molécula, como son: aniónicos que en una solución acuosa se disociarán en un anión anfífilo y un catión que generalmente son metales alcalinos o un amino cuaternario (Salager, 2002). Los no iónicos en una solución acuosa no forman iones, porque su parte hidrofílica es formada por grupos polares que no estén ionizados, estos se solubilizan por un efecto mezclado de un grupo de solubilizantes débiles llamados hidrófilos como grupos funcionales que tienen en su molécula enlaces tipo éter o grupos hidroxilos (Espinoza, 2017). Los catiónicos se disocian en un catión anfífilo y un anión; se los utilizan en ciertas ocasiones cuando sea necesario que la carga positiva de 
ventajas como en una emulsión asfáltica, la mayoría de estos son compuestos nitrogenados con amina grasa o sal de amonio cuaternario. La producción de estos agentes tensioactivos es más cara que los demás (Sanz, 2015). Los anfotéricos son combinados con un grupo aniónico y otro grupo catiónico, en estos surfactantes el pH determina el lado dominante una posible disociación es: Aniónico a pH alcalino, Catiónico a pH ácido. La mayoría de este tipo de surfactantes tienen grupos funcionales tipo amina o amonio, pero pueden ser bloqueado por una cuaternización (Salager y Fernández, A., 2004).

Los surfactantes afectan la tensión superficial entre un fluido y un gas porque sus moléculas debilitan las fuerzas existentes del fluido. Dentro del fluido, existen fuerzas de interacción molecular. En el interior del fluido la fuerza resultante entre moléculas es nula, y estas se encuentran en equilibrio, mientras que las moléculas en la cercanía de la superficie estarán sometidas a una fuerza resultante con dirección al interior. Finalmente, las moléculas situadas en la superficie del fluido, tendrán una fuerza dirigida hacia el interior, pero con mayor magnitud que en el caso de la cercanía a la superficie. (Franco, 2009).

Al momento que se añade surfactante a un fluido, este empieza a adsorberse a las interfaces disponibles, después su concentración en forma monomolecular aumenta hasta el punto que forman las primeras micelas (Salager, 1992). Las moléculas del surfactante van formando grupos que, en una disolución acuosa o polar, las moléculas anfifílicas forman micelas en los grupos polares que están en la superficie y las partes apolares quedan inmersas en el interior de la micela, de esta manera se eliminan los contactos entre el agua y las zonas hidrófobas, permitiendo así la solvatación de los grupos polares. Pero en un medio apolar, las moléculas anfifílicas forman micelas en los grupos apolares, que se encuentran en la superficie y las partes polares quedan inmersas en el interior de la micela, eliminando el contacto entre el medio apolar y la zona hidrofílica (Carrero y Herráez, 2012), como se muestra en la Figura 3.

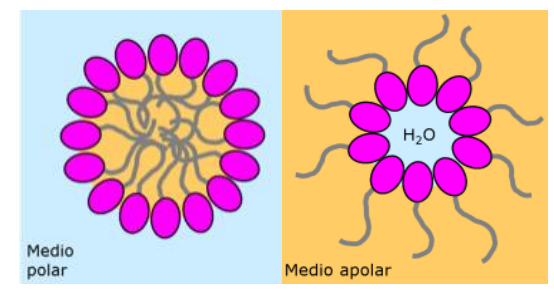

Figura 3. Formación de Micelas medio Polar y apolar (Fuente: Carrero, I., Herráez, A., 2012)

La unión de una cantidad mínima de moléculas de un surfactante se la conoce como la concentración micelar crítica (CMC), esta concentración mínima es requerida para la formación de micelas en una disolución como se observa en la Figura 3. Esta CMC puede ser medida de diferentes maneras, como: resonancia magnética, medición de la conductividad, o por la medición de la tensión superficial (Carrero y Herráez, 2012). La CMC es una de las medidas más importantes de los surfactantes ya que la tensión superficial o interfacial depende directamente de la concentración del surfactante. Por debajo de la CMC la conductividad y la tensión superficial e interfacial aumentan mientras que sobre la CMC la conductividad va a ser menor, las tensiones tanto superficial como interfacial van a llegar a un punto constante, la cantidad de micelas aumenta, lo que es perjudicial debido a que se puede formar emulsiones. (Novelo y Fadrique, 2005).

Los surfactantes también afectan a la tensión interfacial (IFT) entre dos líquidos, también conocida como la energía libre de Gibbs por unidad de área de la interfaz, pero depende de la temperatura y de la presión. Este es el resultado de la interacción de dos diferentes moléculas, es decir la molécula la cual está cercana a la interfaz es diferente que la del otro fluido (Schlumberger, $2018 \mathrm{c}$ ).

Este trabajo se centró en la medición de la tensión, tanto superficial como interfacial, por el método del anillo Du Noüy; que es el reemplazo de la placa rectangular de Wilhelmy ya que posee menos superficie de contacto al momento de interactuar con el líquido a analizar. El anillo debe tener características conocidas como el radio y su peso, de preferencia el anillo debe estar fabricado de platino o una aleación de iridio - platino (Salager, 2005).

\subsection{Espectrometría Infrarroja (Infrared Spectroscopy)}

La espectrometría infrarroja (IR) es utilizada para estudiar muestras con independencia de su estado ya sea que se encuentren como: líquidos, disoluciones, pastas, polvos, fibras, films, gases o superficies. (Serrano, 2009). El análisis IR analiza cualitativamente y cuantitativamente los grupos funcionales presentes en el material a analizar (Piqué y Vázquez, 2012.).

La espectrometría infrarroja estudia la absorción o la emisión de energía radiante originada por la interacción entre la radiación y el material a estudiar. Esta se basa en los modos normales de vibración a distintas frecuencias (Piqué y Vázquez, 2012). Es decir, al momento que una molécula absorbe energía de un haz de luz infrarroja se da una determinada transición vibracional. Se pueden distinguir dos vibraciones específicas, como: vibraciones de tensión que son los cambios en la distancia a lo largo de un enlace covalente y las vibraciones de flexión que van a originar el cambio de ángulo (EHUS, 2006).

Existe un rango de regiones de frecuencias del infrarrojo, desde los límites de $0.8 \mu \mathrm{m}$ a $1000 \mu \mathrm{m}$ que corresponden desde 12800 a $10 \mathrm{~cm}^{-1}$ según sus longitudes de onda las cuales son: El I.R. Cercano, el Fundamental o Medio y el Lejano (U.A. Chihuahua, 2012), como muestra la Figura 4.

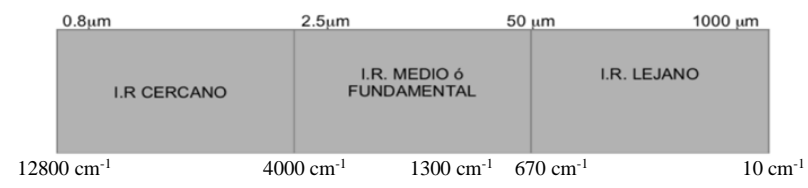

Figura 4. Regiones del Análisis IR

La mayoría de las sustancias a analizar se encuentran situadas en la región IR Medio o fundamental y dentro de esta región se subdivide en dos regiones más las que son: Grupos funcionales en los rangos de $4000 \mathrm{~cm}^{-1}$ a $1300 \mathrm{~cm}^{-1}$ y región dactilar en los rangos de $1300 \mathrm{~cm}^{-1}$ a $670 \mathrm{~cm}^{-1}$ (U.A. Chihuahua, 2012). 
Un espectro infrarrojo se puede manifestar en bandas de absorción. Cada una de estas bandas corresponden a un movimiento vibracional específico de un enlace molecular dentro del compuesto (Piqué y Vázquez, 2012). Es decir, cada compuesto tendrá un comportamiento característico frente a un haz de luz infrarrojo, como una huella dactilar, y se situará en una banda respectiva.

La Figura 5 muestra las bandas de absorción que se encuentran dentro de una gráfica, que en el eje de las ordenadas es la transmitancia $(\% \mathrm{~T})$ que es la cantidad de luz que atraviesa un cuerpo (González, 2010), y el eje de las abscisas representa las longitudes de onda $\left(\mathrm{cm}^{-1}\right)$. La Tabla 2 indica la frecuencia y el tipo de vibración de algunos enlaces.

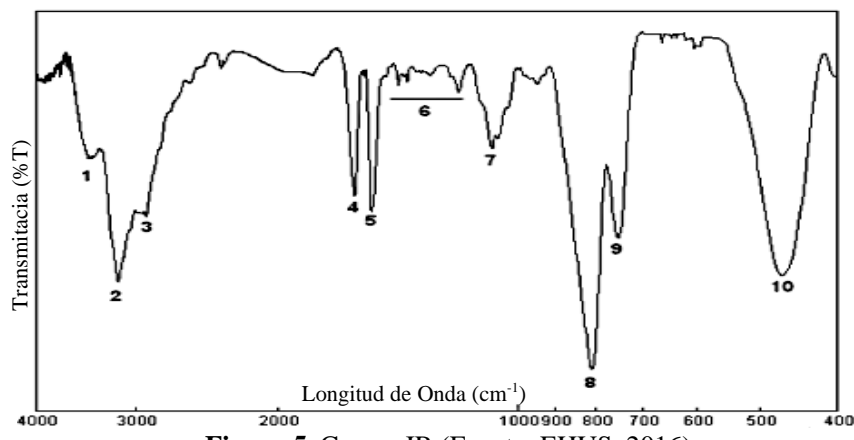

Figura 5. Curvas IR (Fuente: EHUS, 2016)

Tabla 2. Frecuencia y tipo de vibración (Fuente: EHUS, 2016)

\begin{tabular}{cccc}
\hline $\begin{array}{c}\text { Pico de la } \\
\text { figura }\end{array}$ & $\begin{array}{c}\text { Longitud de onda } \\
\left(\mathbf{c m}^{-1}\right)\end{array}$ & Enlace & Tipo de vibración \\
\hline 1 & 3450 & $\mathrm{O}-\mathrm{H}$ & Tensión \\
2 & 3170 & $\mathrm{~N}-\mathrm{H}$ & Tensión \\
3 & 2950 & $\mathrm{C}-\mathrm{H}$ & Tensión \\
4 & 1610 & $\mathrm{O}-\mathrm{H}$ & Flexión \\
5 & 1535 & $\mathrm{~N}-\mathrm{H}$ & Flexión \\
6 & $1420,1295,1200$ & $\mathrm{C}-\mathrm{H}$ & Flexión \\
7 & 1085 & $\mathrm{C}-\mathrm{N}$ & Flexión \\
8 & 820 & As-O & Tensión (simétrica) \\
9 & 760 & As-O & Tensión (anti simétrica) \\
10 & 470 & As-O & Flexión \\
\hline
\end{tabular}

\section{METODOLOGÍA}

La metodología aplicada es experimental, los ensayos de las muestras se realizaron en el laboratorio de Petróleos de la Universidad Central del Ecuador, a condiciones que para el caso de Quito son $68^{\circ} \mathrm{F}$ y $0,708 \mathrm{~atm}$. Con las diferentes gráficas realizadas se comparó cada una de sus curvas obtenidas y de esta manera se analizó cuál, de los surfactantes utilizados, presentó una mayor disminución de tensiones. Tomando en cuenta las gráficas CMC, IFT y TS Vs Concentración.

La primera fase del trabajo consistió en conocer las características de los fluidos a utilizar, como el \% BSW en las tres muestras de petróleo de los pozos PIN 11, 13 y 17, y la salinidad y los sólidos disueltos del agua de inyección (TDS). La medición del BSW se lo realizó con la norma ASTM D40007-02. Se prepararon cuatro zanahorias agregando petróleo, Tolueno y un solvente de enzimas (Greenzyme), luego a las zanahorias se las agitó, se las calentó y se las colocó en la centrifuga por al menos 20 minutos. Posteriormente, se leyó la cantidad de BSW de cada una de las zanahorias y se calculó con la ecuación (1), fue necesario multiplicar por 2, debido a que se usó $50 \mathrm{ml}$ de soluciones.

$$
\% B S W=\frac{\text { Cantidad de solidos y de agua }}{\text { Cantidad Total de muestra de petroleo }} \times 100 \times 2
$$

La medición de la salinidad y el TDS presente en el agua de inyección, se midió con un conductímetro. Se colocó $20 \mathrm{ml}$ de agua de inyección en un cristalizador, posteriormente se colocó el conductímetro, para así obtener las propiedades del agua de inyección.

La segunda fase consistió en la calibración del tensiómetro marca DATAPHYSIC DCAT11EC para determinar la precisión en las mediciones a realizar. Se midió la tensión superficial experimental y se comparó con la tensión superficial teórica de cuatro sustancias conocidas. Se utilizó el anillo Du Noüy fabricado de una aleación de Iridio-Platino. Para la calibración se colocó cierta cantidad de Tolueno, Agua Destilada Tipo 2, Agua Potable y Metanol en cuatro diferentes cristalizadores, estos fueron llevados al tensiómetro y se procedió a la medición de la tensión superficial; para cada muestra se realizaron 7 veces las mediciones y se obtuvo un valor promedio. En la Tabla 3 se muestran los resultados obtenidos de cada una de las sustancias.

Tabla 3. Calibración Tensión Superficial

\begin{tabular}{lccccccc}
\hline \multicolumn{2}{c}{ Tolueno } & \multicolumn{2}{c}{ Agua dest. tipo 2 } & \multicolumn{2}{c}{ Agua } & \multicolumn{2}{c}{ Metanol } \\
\hline Promedio & 25.777 & Promedio & 70.349 & Promedio & 70.9735 & Promedio & 27.92 \\
$\begin{array}{l}\text { Valor } \\
\text { Teórico }\end{array}$ & 28.5 & $\begin{array}{c}\text { Valor } \\
\text { Teórico }\end{array}$ & 72.75 & $\begin{array}{c}\text { Valor } \\
\text { Teórico }\end{array}$ & 72.75 & $\begin{array}{c}\text { Valor } \\
\text { Teórico }\end{array}$ & 22.61 \\
$\begin{array}{l}\text { Error } \\
\text { ABS }\end{array}$ & 2.7225 & $\begin{array}{c}\text { Error } \\
\text { ABS \% }\end{array}$ & 2.4002 & $\begin{array}{c}\text { Error } \\
\text { ABS }\end{array}$ & 1.77642 & $\begin{array}{c}\text { Error } \\
\text { ABS }\end{array}$ & 5.31 \\
\hline
\end{tabular}

En la Tabla 3, se puede observar los diferentes valores de errores absolutos. La temperatura tiene un papel muy importante, debido a que la TS disminuye a medida que la temperatura aumenta (Mak, 1990). La temperatura de la ciudad de Quito es variable y esta puede subir hasta $24.5^{\circ} \mathrm{C}$ (INAMHI, 2019). Otro factor que interfiere en las mediciones es la pureza de los fluidos a analizar, los fluidos utilizados como el Tolueno, Metanol y el agua destilada tipo 2, no son sustancian puras. El agua, es el fluido que más se acerca a la medición teórica, esto se debe a que esta sustancia es de fácil obtención. Un dato significativo sobre el anillo Du Noüy, es que presente una soldadura con el mismo material de fabricación (aleación de iridio con platino), y el equipo fue calibrado exitosamente para su correcto funcionamiento.

La tercera fase consistió en realizar mediciones del pH de cada uno de los surfactantes y realizar mezclas de agua de inyección a diferentes concentraciones para obtener valores de conductividad y tensión superficial. Para realizar las mediciones se colocó en un cristalizador una cantidad de agua de inyección más surfactante a diferentes concentraciones; primero se colocó en el conductímetro para obtener la medida de la conductividad, posterior al tensiómetro para obtener las medidas de TS y así generar las diferentes curvas de TS Vs Concentración, para conocer su CMC. Las mediciones de $\mathrm{pH}$ en la Tabla 4. 
Tabla 4. $\mathrm{pH}$ - Surfactantes

\begin{tabular}{cccccc} 
& & & \multicolumn{3}{c}{ pH agua de formación } \\
\cline { 3 - 6 } & & & \multicolumn{3}{c}{} \\
\hline NH & TEMP. & PIN 11 & PIN 13 & PIN 17 \\
\hline HALMX152805-2 & 7.42 & 22 & & & \\
HALMX152805-1 & 6.65 & 21.8 & 6.21 & 6.25 & 7.00 \\
BH - WCW 2827 & 7.17 & 21.7 & & & \\
BH - WAW 3037 & 12.25 & 21.8 & & & \\
\hline
\end{tabular}

La medición del pH se lo realiza con el objetivo de conocer si el surfactante inyectado va a ser compatible con el agua de formación dentro del pozo. Siendo uno de los parámetros para conocer la calidad del agua con surfactante a inyectarse, se lo realiza para evitar daños potenciales en el pozo como la formación de costras insolubles, emulsiones, la precipitación de ceras y asfáltenos, la reacción con las arcillas dentro del pozo y para evitar corrosión en las tuberías (Naranjo, et al, 2010). Como se puede observar en la Tabla 4 los valores de $\mathrm{pH}$ de surfactante con los valores de $\mathrm{pH}$ del agua de formación entran en un rango similar. Exceptuando el surfactante BakerHughs WAW-3937, el valor de 12.25 indica que es alcalino.

La cuarta fase consistió en la generación de curvas de IFT Vs. Concentración; para esto se preparó en un cristalizador una mezcla de agua de inyección con surfactante a diferentes concentraciones, y en otro cristalizador se colocó petróleo. Primero se colocó la muestra de petróleo en el tensiómetro para medir la fuerza que ejerce el líquido más liviano, después se puso la mezcla de agua de inyección con surfactante en el tensiómetro con el anillo sumergido. Mediante una jeringa de vidrio, se inyectó una cantidad de petróleo sobre esta mezcla para generar la interfaz y con el tensiómetro medir la IFT.

Finalmente, las muestras de surfactante fueron llevadas al laboratorio de Química de la Universidad Central del Ecuador para realizar un análisis IR e identificar los grupos funcionales de cada uno de los surfactantes. Con los datos y curvas obtenidas se analiza para decidir sobre el surfactante, en que concentración y el grupo funcional que actuó mejor.

\section{RESULTADOS Y DISCUSIÓN}

\subsection{Propiedades del agua y BSW del petróleo}

Es importante caracterizar los fluidos que se van a analizar, razón por la cual se obtuvo el \% BSW y el ${ }^{\circ} \mathrm{API}$ del petróleo de los pozos PIN 11,13 y 17. También, la salinidad y los sólidos disueltos en el agua de inyección. Los resultados obtenidos se encuentran en la Tabla 5.

Tabla 5. Propiedades de Agua de Inyección (A) y de Petróleo del campo Pindo (B)

\begin{tabular}{cc}
\hline \multicolumn{2}{c}{$\begin{array}{c}\text { Propiedades del Agua de } \\
\text { Inyección }\end{array}$} \\
\hline Salinidad & $65,7 \mathrm{PPM}$ \\
TDS & $98,6 \mathrm{~g} / 1$ \\
\hline
\end{tabular}

(A)

\begin{tabular}{cccc}
\hline $\begin{array}{c}\text { Pozos } \\
\text { PIN }\end{array}$ & $\begin{array}{c}\text { BSW } \\
(\%)\end{array}$ & ${ }^{\circ}$ API & $\begin{array}{c}\text { BSW } \\
\text { del } \\
\text { campo }\end{array}$ \\
\hline 11 & 4 & 17.9 & \\
13 & 6.8 & 17.3 & $5 \%$ \\
17 & 1.6 & 19.5 & \\
\hline
\end{tabular}

(B)

\subsection{Tensiones Superficiales y Conductividad}

Se generaron gráficas para poder analizar el comportamiento de la conductividad y tensión superficial. La Figura 6 contiene las curvas de conductividad Vs log concentración y la Figura 7 contiene las curvas de TS Vs log de la concentración. Para una mejor visualización, el eje de las abscisas está como logaritmo de la concentración.

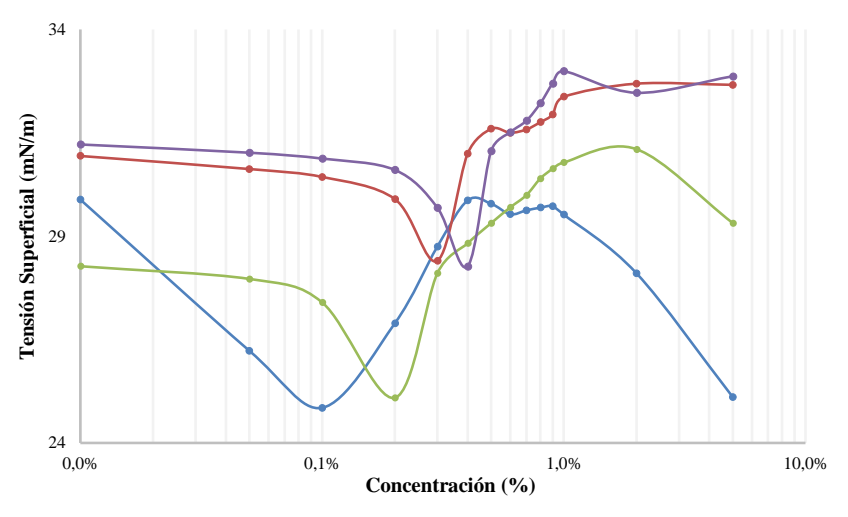

$\longrightarrow$ HALMX152805-2 — HALMX152805-1 — BH - WCW2827 — BH -WAW3037

Figura 6. Tensión Superficial Vs log Concentración

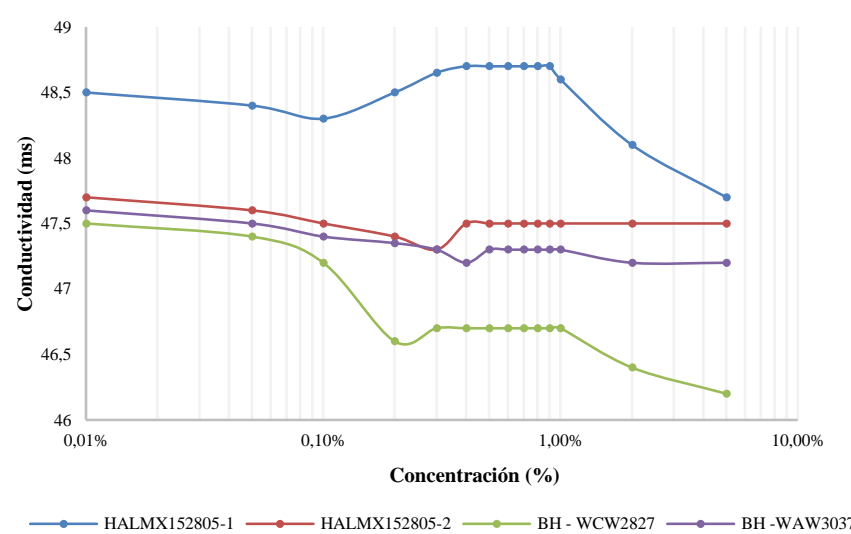

Figura 7. Conductividad Vs log Concentración

La CMC se obtuvo con los resultados de la TS y de la conductividad. Se realizaron 15 muestras a diferentes concentraciones para cada surfactante, que van desde $0 \%$ hasta el $5 \%$. El porcentaje de concentración y el resultado tanto de conductividad y TS se presentan en los anexos en las tablas 12 y 13. Con estas tablas se prepararon las Figuras 6 y 7 .

Las Figuras 6 y 7 contienen las curvas de todas las mediciones de la TS y conductividad a diferentes concentraciones de surfactantes. Analizando, en la Figura 6 se observa que cada curva llega hasta punto de deflexión, dicho punto es el valor de CMC para cada surfactante. Luego, comienza a aumentar hasta tener un comportamiento constante. La Figura 7 tiene el mismo comportamiento; es decir, llega hasta un punto de deflexión y luego un aumento hasta tener un comportamiento constante. Esto se debe al comportamiento intermolecular, ya que el surfactante tiene como objetivo debilitar las fuerzas intermoleculares y el campo eléctrico intermolecular (Salager, 1992). El comportamiento constante de las gráficas llega hasta el $1 \%$, después de este punto, las curvas empiezan a disminuir esto se debe a que existe una saturación de micelas y hay un comportamiento no-ideal, ya que se puede formar una emulsión y en casos extremos se pueden obtener estructuras tipo gel o de cristal líquido (Salager, 1993). Los valores de CMC de cada uno de las muestras se encuentran en la Tabla 6. 
Tabla 6. Resultados T.S y Conductividad

\begin{tabular}{cccc}
\hline Surfactante & CMC (\%) & T.S (mN/m) & Cond. (ms) \\
\hline HALMX152805-2 & 0,10 & 24,849 & 48,3 \\
HALMX152805-1 & 0,30 & 28,407 & 47,3 \\
BH WCW2827 & 0,20 & 25,092 & 46,6 \\
BH WAW3037 & 0.40 & 28,270 & 47,2 \\
\hline
\end{tabular}

Los surfactantes HALMX152805-2 y BH WCW2827 dieron buenos resultados en la disminución de la TS como se muestra en la Tabla 10, siendo el mejor el HALMX152805-2.

\subsection{Tensiones Interfaciales}

Se generaron gráficas a partir de los resultados obtenidos, con el objetivo de analizar el comportamiento de la IFT a diferentes concentraciones para cada pozo. Las Figuras 8, 9 y 10 son las gráficas de IFT Vs Log concentración de los pozos PIN 11,13 y 17.

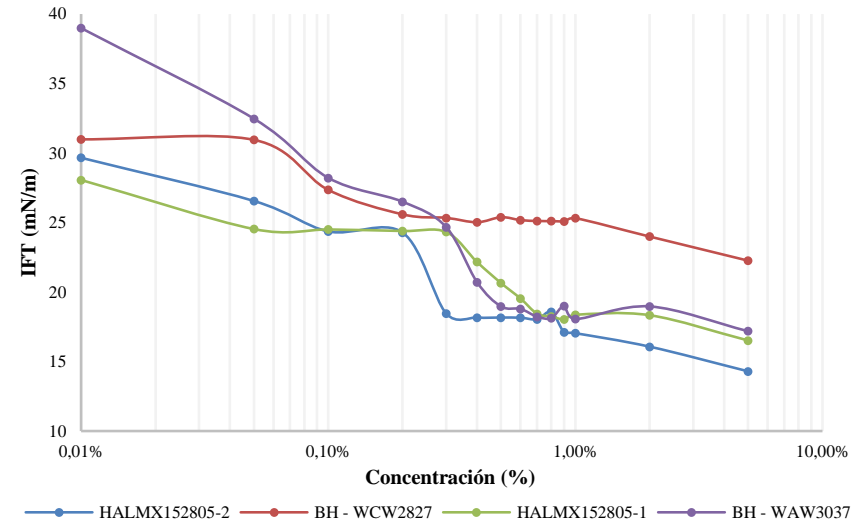

Figura 8. Tensiones Interfaciales Vs. Log Concentración Campo Pindo 11

La Figura 8 muestra las curvas de IFT del pozo PIN 11, estas presentan diferentes puntos de deflexión y luego tienden a mantenerse constante en sus valores, también se puede observar, el surfactante que tiene la reducción más significativa es el HALMX152805-2, el cual tiene una tensión interfacial de 18,46 al $03 \%$.

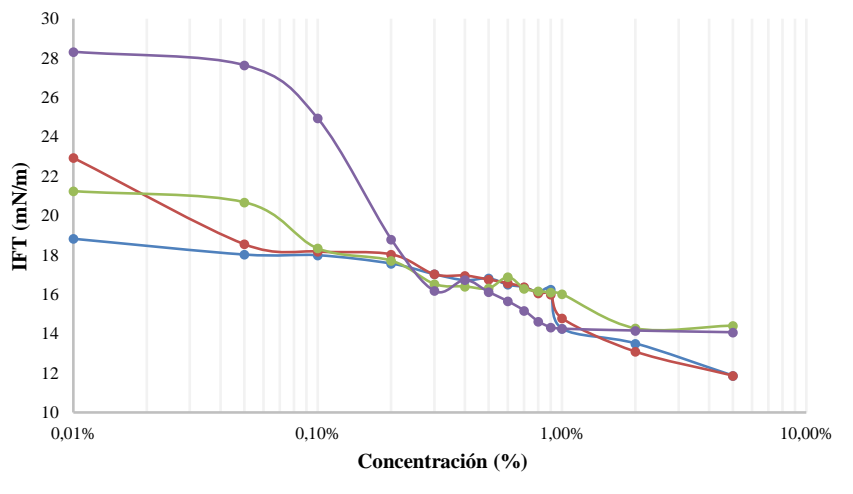

Figura 9. Tensiones Interfaciales Vs. Log Concentración Campo Pindo 13

La Figura 9 muestra las curvas de IFT del pozo PIN 13, presentando diferentes puntos de deflexión y luego tienden a mantenerse constante en sus valores, también se puede observar, que el surfactante Baker Hughes WAW3037 tiene la reducción más significativa con una tensión interfacial de 16,179 al 03\%. Pero, el HALMX152805-1 también muestra un descenso significativo de la IFT con 16,531 al 0,3\%.

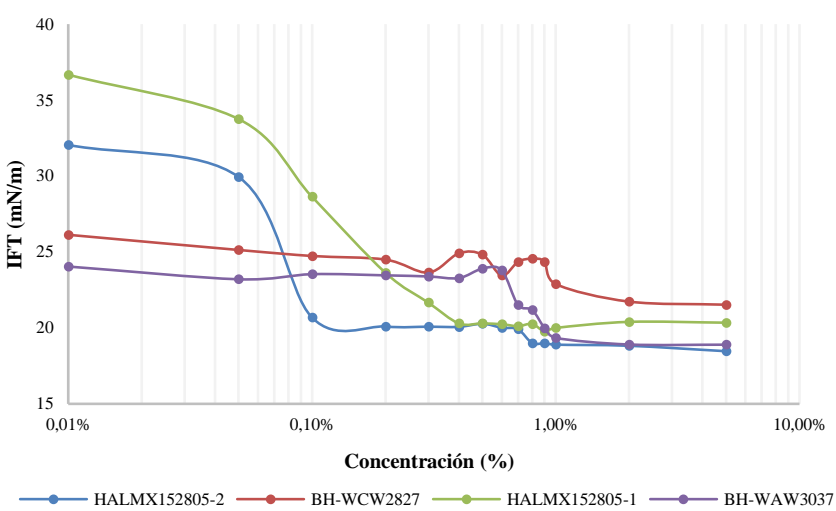

Figura 10. Tensiones Interfaciales Vs. Log Concentración Campo Pindo 17

La Figura 10 muestra las curvas de IFT del campo PIN 17, las curvas presentan diferentes puntos de deflexión y posteriormente tienden a mantenerse constantes. También, se puede observar que el surfactante HALMX1528005-2 tiene una reducción más significativa de IFT, con una medición de 20,688 al $0,1 \%$ de concentración.

El análisis de la tensión interfacial (IFT) empieza desde las mediciones realizadas de CMC; la medición de la CMC se la realiza para obtener la mínima concentración de surfactante para la generación de micelas, por encima de la CMC no se va absorbiendo moléculas adicionales en la fase o interfase (Hernández, 2004). Al momento de introducir concentraciones altas pasadas de la CMC existe una saturación de micelas llevando a un comportamiento no-ideal, ya que se puede formar una emulsión y en casos extremos se puede obtener estructuras tipo gel o de cristal líquido (Salager, 1993). Razón por la cual las concentraciones deben coincidir o estar cerca de la CMC de cada surfactante.

Para seleccionar las mejores concentraciones de cada surfactante se analizó el punto de deflexión en las curvas de IFT y se la corroboró con la CMC, debido a que debe coincidir o ser cercano a este valor. Las mejores concentraciones son bajas y esto también es uno de los aspectos que se debe tomar en cuenta por el factor económico ya que es un trabajo de recuperación mejorada se debe inyectar continuamente surfactante; por esta razón, en la mayoría de los proyectos, se han utilizado bajas concentraciones de surfactante (Chuck y Trombetta, 2007).

La Tabla 7 muestra las concentraciones cuando las curvas se encuentran en los diferentes puntos de deflexión. De los valores presentados en la tabla, se selecciona el HALMX152805-2; debido a que en dos de los tres pozos presentó la mejor reducción de IFT a menor o igual concentración. Otro aspecto de su selección es por la CMC, como se puede observar en Tabla 7, tiene la mejor reducción de la TS a menor concentración. El único caso donde la IFT tiene un resultado diferente es para el pozo Pin 13, los surfactantes Baker Hughes WAW3037 y HALMX152805-1, 
proporcionaron buenos resultado. Pero las mediciones de TS y al CMC son mayores que las del HALMX152805-2.

Tabla 7. Resultados IFT

\begin{tabular}{ccccccc}
\cline { 2 - 7 } Surf. & \multicolumn{2}{c}{ PIN 11 } & \multicolumn{2}{c}{ PIN 13 } & \multicolumn{2}{c}{ PIN 17 } \\
\cline { 2 - 7 } & $\begin{array}{c}\text { Conc. } \\
(\boldsymbol{\%})\end{array}$ & $\begin{array}{c}\text { IFT } \\
(\mathbf{m N} / \mathbf{m})\end{array}$ & $\begin{array}{c}\text { Conc. } \\
(\boldsymbol{\%})\end{array}$ & $\begin{array}{c}\text { IFT } \\
(\mathbf{m N} / \mathbf{m})\end{array}$ & $\begin{array}{c}\text { Conc. } \\
(\boldsymbol{\%})\end{array}$ & $\begin{array}{c}\text { IFT } \\
(\mathbf{m N} / \mathbf{m})\end{array}$ \\
\hline HALMX152805-2 & 0,30 & 18,460 & 0,30 & 17,022 & 0,30 & 21,668 \\
HALMX152805-1 & 0,30 & 24,320 & 0,30 & 16,531 & 0,30 & 23,389 \\
BH WCW2827 & 0,30 & 24,678 & 0,30 & 17,023 & 0,30 & 23,647 \\
BH WAW3037 & 0,20 & 25,588 & 0,20 & 18,034 & 0,10 & 23,539 \\
\hline
\end{tabular}

\subsection{Espectrometría infrarroja (IR)}

Los surfactantes fueron enviados al laboratorio de la Universidad Central del Ecuador, para que realicen un análisis de espectrometría infrarroja y así, obtener los grupos funcionales de cada uno de los surfactantes. Las Tablas 8, 9, 10 y 11 , muestran las longitudes de onda de cada uno de los surfactantes.

Tabla 8. Grupo Funcional - HALMX152805-1

\begin{tabular}{cc}
\hline $\begin{array}{c}\text { Grupo } \\
\text { Funcional }\end{array}$ & Longitud de Onda \\
\hline Grupo Alquil & $3820-3200 / / 2980-2830 / / 1480-1430$ \\
Compuesto & $3650-3590$ \\
Hidroxilo & \\
Grupo & $3400-3150 / / 3000-2850 / / 2800-2500 / / 1880-$ \\
Carbonilo & $1670 / / 1570-1520 / / 1300-1250$ \\
\hline
\end{tabular}

Según los valores de longitud de onda de la Tabla 8, que pertenece al surfactante HLAMX152805-1, podemos analizar cada una de las bandas de los grupos funcionales. El grupo alquil está trabajando en las bandas $1,2,3,4,5$ y 6 ; el grupo hidroxilo está en las bandas 1 y 2 ; y el grupo carbonilo está en las bandas 2, 3, 4, 5 y 6 .

Tabla 9. Grupo Funcional - HALMX152805-2

\begin{tabular}{cc}
\hline $\begin{array}{c}\text { Grupo } \\
\text { Funcional }\end{array}$ & Longitud de Onda \\
\hline Grupo Alquil & $3820-3200 / / 2980-2830 / / 1480-1430$ \\
Compuesto & $3650-3590 / / 2300-2000$ \\
Hidroxilo & \\
\hline
\end{tabular}

Según los valores de longitud de onda de la Tabla 9, que pertenece al surfactante HLAMX152805-2, podemos analizar cada una de las bandas de los grupos funcionales. El grupo alquil está trabajando en las bandas $1,2,3,4,5$ y 6 ; el grupo hidroxilo está en las bandas 1, 2 y 3 .

Tabla 10. Grupo Funcional - BAKER HUGHES WCW 2827

\begin{tabular}{cc}
\hline $\begin{array}{c}\text { Grupo } \\
\text { Funcional }\end{array}$ & Longitud de Onda \\
\hline Grupo Alquil & $3820-3200 / / 2980-2830 / / 1480-1430$ \\
Compuesto & $3650-3590$ \\
Hidroxilo & $3400-3150 / / 3000-2850 / / 2800-2500 / / 1880-$ \\
Grupo & $1670 / / 1570-1520 / / 1300-1250$ \\
Carbonilo & \\
\hline
\end{tabular}

Según los valores de longitud de onda de la Tabla 10, que pertenece al surfactante Baker Hughes WCW 2827, podemos analizar que tienen valores similares a los del componente HLAMX1528; es decir, se encuentra en las mismas bandas de absorción.

Tabla 11. Grupo Funcional - BAKER HUGHES WAW 3037

$\left.\begin{array}{cc}\hline \begin{array}{c}\text { Grupo } \\ \text { Funcional }\end{array} & \text { Longitud de Onda } \\ \hline \text { Grupo Alquilo } & 3540-3200 / / 1205-885 \\ \text { Grupo Alquil } \\ \text { posible Hidroxi } \\ \text { o Amino } \\ \text { sustituido }\end{array}\right]$

Según los valores de longitud de onda de la Tabla 11, que pertenece al surfactante Baker Hughes WAW 3037, podemos analizar cada una de las bandas de los grupos funcionales. El grupo alquil está trabajando en las bandas 1, 2, 6, 7 y 8 ; y el grupo hidroxi o amino está en las bandas 1, 2 y 3 .

También, se observa que los 4 surfactantes se encuentran en el rango del IR medio.

Los surfactantes HALMX152805-1 y HALMX152805-2, poseen grupos funcionales como el Alquil, Compuestos hidroxilo y el Grupo Carbonilo, que pertenecen a surfactantes no iónicos, mientras que los surfactantes Baker Hughes WCW2827 y el Baker Hughes WAW3037 tiene el grupo funcional alquil con compuesto hidroxilo, y con posibilidades de contener Hidroxi o amino sustituido; estos surfactantes son de tipo catiónico.

\subsection{Resultados de medir tensión interfacial y tensión superficial}

El surfactante HALMX152805-2 de tipo no iónico, tiene una menor CMC y presenta resultados de IFT bajos para los pozos PIN 11 y 17; y en el pozo PIN 13 también tiene una reducción significativa de la IFT de 17,022 a 0,3\% en concentración. Este surfactante tiene el grupo funcional Alquil y Compuestos hidroxilo, su CMC fue del $0,1 \%$ y la concentración de $0,3 \%$ dio en los 3 pozos su punto de deflexión.

Otro surfactante que se puede considerar es el BAKER HUGHS WCW2827 de tipo catiónico, con un valor de CMC del $0,2 \%$ y tuvo valores bajos en la reducción de la IFT de los 3 pozos del campo Pindo, el mejor resultado de IFT obtenido fue para el pozo PIN 13. Sus grupos funcionales son el Grupo Alquil, Compuesto hidroxilo y Grupo Carbonilo. Se recomienda para los trabajos de inyección de surfactante, el uso de una concentración de $0,2 \%$ a $0,3 \%$ sobre la base de realizar un promedio de las respuestas de los diferentes pozos.

En la industria petrolera es importante el factor económico, esta es una de las razones del uso de inyección de químicos para sustituir la recuperación térmica (Chuck y Trombetta, 2007). Se recomienda para los trabajos de inyección de surfactante, el uso de una concentración de $0,2 \%$ a $0,3 \%$ por un promedio realizado a las respuestas de concentración. 


\section{CONCLUSIONES}

Del análisis de la CMC y de IFT los mejores surfactantes son el HALMX152805-2 tipo no iónico y el BAKER HUGHES WCW2827 de tipo catiónico; presentaron excelentes resultados para los 3 pozos, pero el de mejor resultados fue el HALMX152805-2.

Las figuras de TS y conductividad muestran un comportamiento similar aspecto que sirve para corroborar que las mediciones están correctamente realizadas y la CMC seleccionada es el adecuado.

La presión y temperatura son factores muy importantes y es fundamental tomar en cuenta las condiciones a realizar los ensayos de laboratorio. Se realizó un pequeño experimento con una mezcla entre de petróleo, agua de inyección y una concentración del surfactante Baker Hughes WCW 2827, la cual arrojó tensiones superficiales e interfaciales más reducidas y se concluye que la IFT es directamente proporcional a la temperatura.

Los análisis de espectrometría infrarroja realizados muestran que los surfactantes usan grupos funcionales parecidos como el Alquil y el Hidroxi.

Con base en los resultados obtenidos se visualiza la disminución de la tensión interfacial y superficial, por lo tanto teóricamente, la disminución de estos dos factores tendrá un resultado en el aumento del flujo dentro del reservorio y una disminución de las presiones capilares.

El pH del agua de formación con el pH de los surfactantes se encuentra en un rango en el cual existe una compatibilidad, lo cual es fundamental para el uso del mismo.

Los errores existen en ciertas sustancias al momento de realizar la calibración fueron debido a las impurezas de los fluidos utilizados, pero con el $\mathrm{H}_{2} \mathrm{O}$ se puede visualizar que la medición tiene un error mucho menor. La temperatura tiene un papel muy importante debido a que en la ciudad de Quito existen cambios repentinos. El anillo Du Noüy fue soldado y calibrado para su correcto funcionamiento.

\section{REFERENCIAS}

Abubaker, A., Abdurahman, N., Z, Y. (2015), “An overview of oil production stages: Enhanced Oil Recovery Techniques and Nitrogen Injection" - Universidad Malaysia Pahang - Kuantan - Malaysia.

Almeida, M., Morales J., Maita, F. (2015), "Procesos de recuperación de crudo", Universidad Nacional Experimental Politécnica de la Fuerza Armada Nacional U.N.E.F.A - Escuela de Petróleo, Núcleo Anzoátegui - Venezuela.

Antón, R. (2005), “Tensión Interfacial” Universidad de los andes, facultad de ingeniería - Escuela de ingeniería química - Lab. Formulación, interfaces, Reología y procesos, Mérida - Venezuela.
ASTM (2006), "Método de prueba estándar para agua y sedimentos en petróleo crudo por el método de centrifugación (procedimiento de laboratorio)." designación: D4007-02, An American National Standard.

Bolívar, G., Mantilla, A., Aldás, A., Gonzales, M., Torres, C., (diciembre,2017), "Influencia de surfactantes en la evaluación de la tensión interfacial para una emulsión agua petróleo relacionada al proceso de recuperación mejorada", Universidad Central del Ecuador, Quito Ecuador.

Carrero I. y Herráez (2012), "Micelas", El mundo de los lípidos Biomodel, http://biomodel.uah.es/model2/lip/micelas.htm.

Chuck, N. y Trombetta, J. (2007), "El uso de surfactantes en proyectos de recuperación terciaria" Notas técnicas, Tiorco. Págs. $1-4$.

Consorcio Petrosud Petroriva, (2010), "Mapa estructural del Campo Pindo", Figura 1.

Consorcio Petrosud Petroriva (2018-a), "Reseñas campo Pindo, Propiedades de los fluidos del campo Pindo".

Consorcio Petrosud Petroriva (2018-b), "Propiedades de los Pozos Pindo 11, 13 y 17”, Tabla 1.

Consorcio Petrosud Petroriva, (2018-d) "Promedio de producción pozos Pindo - 11 (A), Pindo - 13 (B), Pindo - 17 (C), “U” inferior”. Figura 4.

EHUS (2006), "Espectroscopia infrarroja (IR), EHUS, http://www.ehu.eus/imacris/PIE06/web/IR.htm\#arrib a.

Espinoza, J., (2017), “Tensioactivos", https://biorem.univie.ac.at/fileadmin/user_upload/p_ biorem/education/lectures/SEMARNAT/SEMARN AT-E-Clasificacion_de_Tensoactivos.pdf

Franco, A. (2009), “Tensión superficial en los líquidos”, Curso interactivo de física, Universidad de Granada, http://www.sc.ehu.es/sbweb/fisica_/fluidos/tension/i ntroduccion/introduccion.html.

González, M. (2010), “Transmitancia y Absorvancia”, La guia de Quimica, https://quimica.laguia2000.com/conceptosbasicos/transmitancia-y-absorbancia.

Hernández, C. (2004), "Formación de nano emulsiones o/w mediante el cambio en la composición - formulación por dilución con agua de sistemas próximos a la formulación óptima", Universidad de los andes, facultad de ingeniería - escuela de ingeniería química, Mérida - Venezuela.

Hernández, G. (2008), "Producción de Petróleo", http://www.gustato.com/petroleo/Petroleo3.html. 
INAMHI, (2019), “Analisis Climatológico" Dirección de gestion meteorologica e invetigación meteorologicas. Quito - Ecuador.

Macho, S., (2002) "Motodologías analíticas basadas en espectroscopia de infrarrojo y calibración multivariante. Aplicación a la industria petroquímica", departamento de química analítica y química organica, Universitat Rovira i Virgili, Tarragona, Cataluña - España.

Mak, S., Wong, K. (1990), "The measurement of the surface tension by the method of direct pull", pp. 791-792, http://www.sc.ehu.es/sbweb/fisica/fluidos/tension/int roduccion/introduccion.htm

Morales, A. (2010), "Inyección de surfactantes en yacimientos", Petróleo América, http://www.petroleoamerica.com/2011/02/inyeccion -de-surfactantes-en.html.

Naranjo, C., Muños, S. Y Zapata, J. (2010), "Factibilidad experimental de la inyección de agua en las arenas mugrosa de campo Lisima”, El reventón Energético.

Novelo, M. y Fadrique, J. (2005), “Concentración Micelar crítica mediante la ecuación de adsorción de Gibbs", Profesores al día (fisicoquímica).

Piqué, T., Vázquez, A., (2012), "uso de espectroscopia infrarroja con transformada de fourier (ftir) en el estudio de la hidratación del cemento" Concreto y cemento. Investigación y desarrollo. http://www.scielo.org.mx/pdf/ccid/v3n2/v3n2a4.pdf

Rodríguez, A. (2015)" Características de los campos Pindo y Palanda Yuca Sur", Escuela Politécnica Nacional, Facultad de ingeniería en Geología y Petróleos, Quito - Ecuador.

Rondón, N. (2009), "Métodos de recuperación mejorada", https://quipu.uni.edu.pe/OtrosWWW/webproof/acad e/fipp/lucioc/EOR107.html

Salager, J. (1992), "El mundo de los surfactantes" Cuaderno FIRP S311-A, Modulo de enseñanza en fenómenos interfaciales, Universidad de los andes, facultad de ingeniería - escuela de ingeniería química, Mérida Venezuela.

Salager, J. (1993), "Surfactantes en solución acuosa" Cuaderno FIRP S2001-A, Modulo de enseñanza en fenómenos interfaciales, Universidad de los andes, facultad de ingeniería - escuela de ingeniería química, Mérida - Venezuela

Salager, J. (2002), "Surfactantes Tipos y Usos" Cuaderno FIRP S300-A, Modulo de enseñanza en fenómenos interfaciales, Universidad de los andes, facultad de ingeniería - escuela de ingeniería química, Mérida Venezuela.
Salager, J. y Fernández, A. (2004), "Surfactantes”, Cuaderno de enseñanza en fenómenos interfaciales, Universidad de los andes, facultad de ingeniería escuela de ingeniería química, Mérida - Venezuela.

Salager, J. (2005), "Recuperación Mejorada del Petróleo", Cuaderno FIRP S357-C módulo de enseñanza en fenómenos interfaciales, Universidad de los andes, facultad de ingeniería - escuela de ingeniería química, Mérida - Venezuela.

Salager, J. y Antón, R. (2005), "Métodos de medición de la tensión Superficial o Interfacial", Cuaderno FIRP N. ${ }^{\circ}$ 205B, Universidad de los andes, facultad de ingeniería - Escuela de Ingeniería Química, Mérida Venezuela

Salager, J., Rojas, O., Bullón, J. (2007), "Fenómenos Interfaciales y surfactantes en la industria petrolera" Universidad de los andes, facultad de ingeniería escuela de ingeniería química, Mérida - Venezuela.

Sanz, A. (2015), "La industria de los agentes tensioactivos", https://www.eii.uva.es/organica/qoi/tema-10.php.

Schlumberger (2018 a), Reservoir Engeneering, Quito, Ecuador.

Schlumberger (2018 b). "EOR - Recuperación mejorada de Petróleo",

https://www.glossary.oilfield.slb.com/es/Terms/e/eo r.aspx? $=1$.

Schlumberger (2018 c), “Tensión Interfacial”, Oilfield Glossary,

https://www.glossary.oilfield.slb.com/es/Terms/i/int erfacial_tension.aspx.

Serrano, L., (2009), "Espectroscopia infrarroja 1fundamentos", Curso Instrumentación y método de análisis químico. https://www.upct.es/ minaeees/espectroscopia_infra rroja.pdf

SFN FLOEGER, (2016), “Geología del Petróleo Sistemas Petrolíferos EOR 101”, Oil - EOR Handbook ESP. Edición 2016.

U.A. Chihuahua (2012), "Espectrometría de absorción en el infrarrojo" Lectura N. ${ }^{\circ} 7$ - Facultad de ciencias químicas - Espectrometría, Universidad Autónoma de Chihuahua, México, págs. 2 - 5.

Villegas, I., (2013), "Estudio de factibilidad técnica para el control de agua en el yacimiento "U" Inferior del campo Pindo, Facultad de Ingeniería en Geología, Minas, Petróleo y Ambiental, Universidad Central del Ecuador, Quito - Ecuador. 


\section{BIOGRAFÍAS}

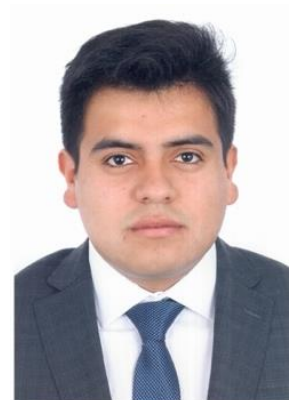

Jonathan Saúl Celi Estévez, bachiller en ciencias generales (Colegio Militar "Eloy Alfaro", 2011). Se graduó de ingeniero en Petróleos de la Escuela Politécnica Nacional (2019). Durante su carrera trabajo en varias empresas como pasante como el Instituto Técnico del Petróleo (ITP) realizando manuales y las compañías Consorcio Petrosud Petroriva y Consorcio Petrolero Palanda Yuca Sur, en la cual desarrollo su tema de tesis y trabajo en laboratorio examinando diferentes muestras. Esta interesado en trabajar en una empresa internacional y seguir dando aportes científicos. Identificador digital ORCID: https://orcid.org/0000-0001-5093-7887

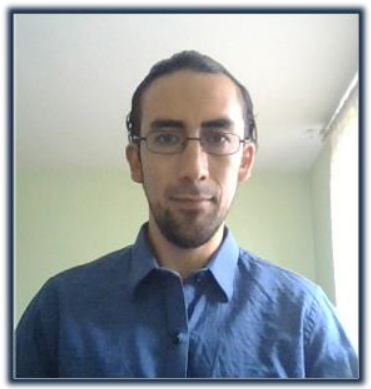

Franklin Vinicio Gómez Soto, ingeniero en Petróleos de la Escuela Politécnica Nacional (EPN, 2010), Asistente de Catedra en el Departamento de Petróleos en la EPN desde el año 2011 hasta el año 2012. Hizo la maestría en Termodinámica de Fluidos en la Universidad de Valladolid, España (UVA, 2012). $\mathrm{Su}$ proyecto de fin de master fue publicado en The Journal of Supercritical Fluids, ELSEVIER. Trabajo como Coordinador Petrolero en Triboil Gas CIA. LTDA durante el año 2013 y 2014. Se reincorporó a la Escuela Politécnica Nacional como docente titular Auxiliar desde octubre del año 2014. Fue jefe del Laboratorio de Fluidos de Perforación y Petrofísica. Actualmente está estudiando su doctorado en la Universidad de Miskolc en Hungría. Se interesa en Fracturamiento Hidráulico y Reacondicionamiento de pozos (reservorios de petróleo y gas). Identificador Digital ORCID: https://orcid.org/0000-0003-4367-4972

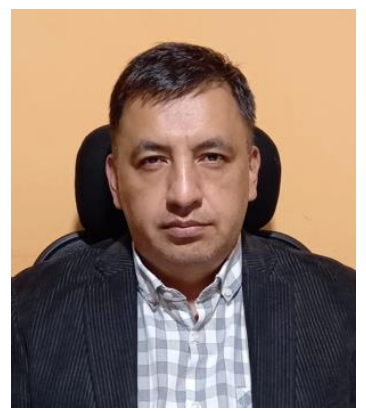

Bolívar Enríquez Vallejo estudió el pregrado en la facultad de ciencias químicas, la carrera de Química, en la Universidad Central del ecuador, posteriormente, realizo una maestría en Sistemas de Gestión de Calidad. Actualmente, se desempeña como director de la Carrera de Ingeniería de petróleos en la misma universidad y adicionalmente, como docente dictando las asignaturas de control de calidad del petróleo y sus derivados, y termodinámica. Dentro de las destrezas más destacadas en él son: capacidad analítica, comunicación asertiva, liderazgo, lo que ha permitido que se desempeñe de manera eficiente en las actividades que ha realizado en su ámbito laboral. Código ORCID: https://orcid.org/00000003-4750-011X

\section{APÉNDICE}

Tablas de Resultados

Usando la ecuación \#1 se procede a calcular BSW en las muestras de petróleo de los 3 pozos

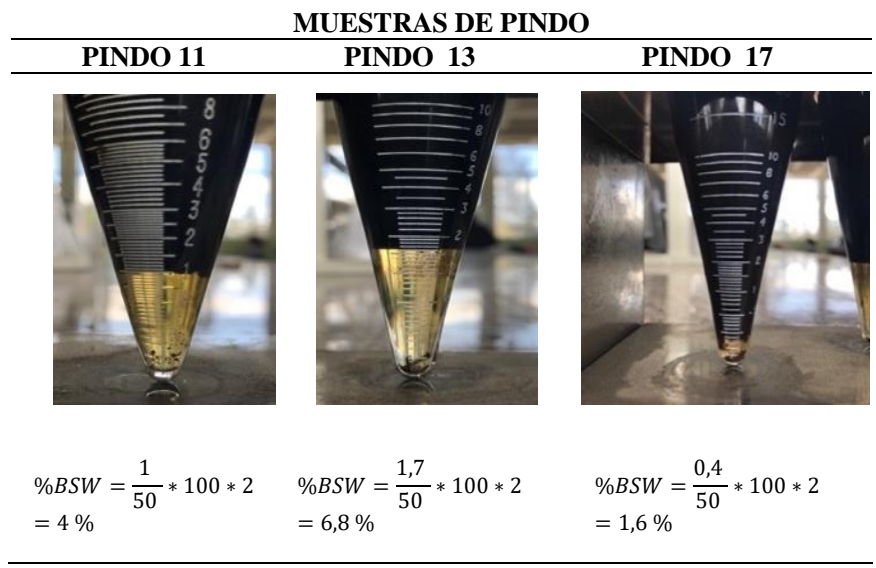

Tablas de Tensiones Superficiales

Tabla 12. Tensiones superficiales y conductividades

\begin{tabular}{|c|c|c|c|c|c|}
\hline \multicolumn{3}{|c|}{ HALMX152805-2 } & \multicolumn{3}{|c|}{ HALMX152805-1 } \\
\hline $\begin{array}{c}\text { Conc } \\
(\%)\end{array}$ & $\begin{array}{c}\text { T.S. } \\
(\mathrm{mN} / \mathrm{m})\end{array}$ & $\begin{array}{c}\text { Cond. } \\
\text { (ms) }\end{array}$ & $\begin{array}{l}\text { Conc } \\
(\%)\end{array}$ & $\begin{array}{c}\text { T.S. } \\
(\mathbf{m N} / \mathbf{m})\end{array}$ & $\begin{array}{c}\text { Cond. } \\
(\mathrm{ms})\end{array}$ \\
\hline $0 \%$ & 45.669 & 48.8 & $0 \%$ & 44.778 & 47.9 \\
\hline $0.01 \%$ & 29.886 & 48.5 & $0.01 \%$ & 30.941 & 47.7 \\
\hline $0.05 \%$ & 26.23 & 48.4 & $0.05 \%$ & 30.625 & 47.6 \\
\hline $0.10 \%$ & 24.849 & 48.3 & $0.10 \%$ & 30.434 & 47.5 \\
\hline $0.20 \%$ & 26.894 & 48.5 & $0.20 \%$ & 29.899 & 47.4 \\
\hline $0.30 \%$ & 28.751 & 48.65 & $0.30 \%$ & 28.407 & 47.3 \\
\hline $0.40 \%$ & 29.865 & 48.7 & $0.40 \%$ & 30.994 & 47.5 \\
\hline $0.50 \%$ & 29.786 & 48.7 & $0.50 \%$ & 31.596 & 47.5 \\
\hline $0.60 \%$ & 29.532 & 48.7 & $0.60 \%$ & 31.503 & 47.5 \\
\hline $0.70 \%$ & 29.625 & 48.7 & $0.70 \%$ & 31.582 & 47.5 \\
\hline $0.80 \%$ & 29.691 & 48.7 & $0.80 \%$ & 31.758 & 47.5 \\
\hline $0.90 \%$ & 29.726 & 48.7 & $0.90 \%$ & 31.945 & 47.5 \\
\hline $1.00 \%$ & 29.523 & 48.6 & $1.00 \%$ & 32.371 & 47.5 \\
\hline $2.00 \%$ & 28.102 & 48.1 & $2.00 \%$ & 32.69 & 47.5 \\
\hline $5.00 \%$ & 25.11 & 47.7 & $5.00 \%$ & 32.661 & 47.5 \\
\hline
\end{tabular}


Tabla 13. Tensiones Superficiales y Conductividades

\begin{tabular}{|c|c|c|c|c|c|}
\hline \multicolumn{3}{|c|}{ BH - WCW2827 } & \multicolumn{3}{|c|}{ BH -WAW3037 } \\
\hline $\begin{array}{c}\text { Conc } \\
(\%)\end{array}$ & $\begin{array}{c}\text { T.S. } \\
(\mathrm{mN} / \mathrm{m})\end{array}$ & $\begin{array}{c}\text { Cond. } \\
\text { (ms) }\end{array}$ & $\begin{array}{c}\text { Conc } \\
(\%)\end{array}$ & $\begin{array}{c}\text { T.S. } \\
(\mathrm{mN} / \mathrm{m})\end{array}$ & $\begin{array}{c}\text { Cond. } \\
\text { (ms) }\end{array}$ \\
\hline $0 \%$ & 32.131 & 47.7 & $0 \%$ & 44.069 & 47.7 \\
\hline $0.01 \%$ & 28.274 & 47.5 & $0.01 \%$ & 31.219 & 47.6 \\
\hline $0.05 \%$ & 27.967 & 47.4 & $0.05 \%$ & 31.02 & 47.5 \\
\hline $0.10 \%$ & 27.4 & 47.2 & $0.10 \%$ & 30.878 & 47.4 \\
\hline $0.20 \%$ & 25.092 & 46.6 & $0.20 \%$ & 30.603 & 47.35 \\
\hline $0.30 \%$ & 28.105 & 46.7 & $0.30 \%$ & 29.688 & 47.3 \\
\hline $0.40 \%$ & 28.828 & 46.7 & $0.40 \%$ & 28.27 & 47.2 \\
\hline $0.50 \%$ & 29.315 & 46.7 & $0.50 \%$ & 31.056 & 47.3 \\
\hline $0.60 \%$ & 29.696 & 46.7 & $0.60 \%$ & 31.513 & 47.3 \\
\hline $0.70 \%$ & 29.992 & 46.7 & $0.70 \%$ & 31.792 & 47.3 \\
\hline $0.80 \%$ & 30.396 & 46.7 & $0.80 \%$ & 32.216 & 47.3 \\
\hline $0.90 \%$ & 30.632 & 46.7 & $0.90 \%$ & 32.698 & 47.3 \\
\hline $1.00 \%$ & 30.785 & 46.7 & $1.00 \%$ & 32.991 & 47.3 \\
\hline $2.00 \%$ & 31.105 & 46.4 & $2.00 \%$ & 32.469 & 47.2 \\
\hline $5.00 \%$ & 29.319 & 46.2 & $5.00 \%$ & 32.869 & 47.2 \\
\hline
\end{tabular}

Tablas de Tensiones Interfaciales

Tabla 14. Tensiones Interfaciales - Pindo 11

\begin{tabular}{ccc|ccc}
\multicolumn{2}{c|}{ BH - WCW2827 } & \multicolumn{3}{|c}{ BH - WAW3037 } \\
\hline $\begin{array}{c}\text { Conc } \\
(\%)\end{array}$ & $\begin{array}{c}\text { IFT } \\
(\mathbf{m N} / \mathbf{m})\end{array}$ & $\begin{array}{c}\mathbf{E} \\
\boldsymbol{+}-\end{array}$ & $\begin{array}{c}\text { Conc } \\
(\%)\end{array}$ & $\begin{array}{c}\text { IFT } \\
(\mathbf{m N} / \mathbf{m})\end{array}$ & $\begin{array}{c}\mathbf{E} \\
\mathbf{+}\end{array}$ \\
\hline $0 \%$ & 49.844 & 1.202 & $0 \%$ & 43.77 & 1.16 \\
$0.01 \%$ & 30.983 & 1.426 & $0.01 \%$ & 38.989 & 1.564 \\
$0.05 \%$ & 30.95 & 1.55 & $0.05 \%$ & 32.474 & 1.738 \\
$0.10 \%$ & 27.351 & 1.505 & $0.10 \%$ & 28.216 & 1.776 \\
$0.20 \%$ & 25.588 & 1.532 & $0.20 \%$ & 26.498 & 1.676 \\
$0.30 \%$ & 25.337 & 1.843 & $0.30 \%$ & 24.678 & 2.097 \\
$0.40 \%$ & 25.022 & 1.844 & $0.40 \%$ & 20.716 & 2.601 \\
$0.50 \%$ & 25.385 & 1.287 & $0.50 \%$ & 18.967 & 3.05 \\
$0.60 \%$ & 25.175 & 0.472 & $0.60 \%$ & 18.788 & 1.994 \\
$0.70 \%$ & 25.115 & 1.489 & $0.70 \%$ & 18.226 & 3.541 \\
$0.80 \%$ & 25.101 & 1.762 & $0.80 \%$ & 18.13 & 2.915 \\
$0.90 \%$ & 25.097 & 1.672 & $0.90 \%$ & 18.994 & 2.967 \\
$1.00 \%$ & 25.315 & 1.807 & $1.00 \%$ & 18.059 & 3.278 \\
$2.00 \%$ & 23.991 & 1.928 & $2.00 \%$ & 18.966 & 2.66 \\
$5.00 \%$ & 22.259 & 2.096 & $5.00 \%$ & 17.184 & 3.29 \\
\hline
\end{tabular}

Tabla 15. Tensiones Interfaciales - Pindo 11

\begin{tabular}{|c|c|c|c|c|c|}
\hline \multicolumn{3}{|c|}{ HALMX152805-1 } & \multicolumn{3}{|c|}{ HALMX152805-2 } \\
\hline $\begin{array}{c}\text { Conc } \\
(\%)\end{array}$ & $\begin{array}{c}\text { IFT } \\
(\mathbf{m N} / \mathbf{m})\end{array}$ & $\begin{array}{l}\mathbf{E} \\
+- \\
\end{array}$ & $\begin{array}{c}\text { Conc } \\
(\%)\end{array}$ & $\begin{array}{c}\text { IFT } \\
(\mathbf{m N} / \mathbf{m})\end{array}$ & $\begin{array}{l}\mathbf{E} \\
+- \\
\end{array}$ \\
\hline $0 \%$ & 51.007 & 1.563 & $0 \%$ & 42.657 & 1.754 \\
\hline $0.01 \%$ & 28.062 & 2.441 & $0.01 \%$ & 29.66 & 2.261 \\
\hline $0.05 \%$ & 24.531 & 2.21 & $0.05 \%$ & 26.554 & 2.049 \\
\hline $0.10 \%$ & 24.502 & 1.797 & $0.10 \%$ & 24.362 & 2.116 \\
\hline $0.20 \%$ & 24.392 & 1.893 & $0.20 \%$ & 24.277 & 1.985 \\
\hline $0.30 \%$ & 24.32 & 2.094 & $0.30 \%$ & 18.46 & 1.851 \\
\hline $0.40 \%$ & 22.162 & 2.374 & $0.40 \%$ & 18.17 & 1.958 \\
\hline $0.50 \%$ & 20.652 & 3.493 & $0.50 \%$ & 18.165 & 1.934 \\
\hline $0.60 \%$ & 19.534 & 2.742 & $0.60 \%$ & 18.154 & 3.065 \\
\hline $0.70 \%$ & 18.441 & 2.589 & $0.70 \%$ & 18.043 & 2.023 \\
\hline $0.80 \%$ & 18.243 & 3.026 & $0.80 \%$ & 18.574 & 3.373 \\
\hline $0.90 \%$ & 18.034 & 2.637 & $0.90 \%$ & 17.112 & 2.076 \\
\hline $1.00 \%$ & 18.363 & 3.222 & $1.00 \%$ & 17.039 & 2.066 \\
\hline $2.00 \%$ & 18.332 & 1.9 & $2.00 \%$ & 16.069 & 2.132 \\
\hline $5.00 \%$ & 16.516 & 1.934 & $5.00 \%$ & 14.297 & 2.089 \\
\hline
\end{tabular}

Tabla16. Tensiones Interfaciales - Pindo 13

\begin{tabular}{|c|c|c|c|c|c|}
\hline \multicolumn{3}{|c|}{ BH - WCW2827 } & \multicolumn{3}{|c|}{ BH - WAW3037 } \\
\hline Conc (\%) & $\begin{array}{c}\text { IFT } \\
(\mathbf{m N} / \mathbf{m})\end{array}$ & $\begin{array}{l}E \\
+-\end{array}$ & $\begin{array}{c}\text { Conc } \\
(\%)\end{array}$ & $\begin{array}{c}\text { IFT } \\
(\mathrm{mN} / \mathrm{m})\end{array}$ & $\begin{array}{l}E \\
+-\end{array}$ \\
\hline $0 \%$ & 22.392 & 1.373 & $0 \%$ & 29.323 & 2.786 \\
\hline $0.01 \%$ & 18.822 & 2.130 & $0.01 \%$ & 28.325 & 1.987 \\
\hline $0.05 \%$ & 18.022 & 2.569 & $0.05 \%$ & 27.645 & 3.543 \\
\hline $0.10 \%$ & 17.989 & 2.978 & $0.10 \%$ & 24.941 & 1.654 \\
\hline $0.20 \%$ & 17.564 & 1.987 & $0.20 \%$ & 18.798 & 3.56 \\
\hline $0.30 \%$ & 17.023 & 2.327 & $0.30 \%$ & 16.179 & 3.789 \\
\hline $0.40 \%$ & 16.719 & 1.819 & $0.40 \%$ & 16.731 & 1.567 \\
\hline $0.50 \%$ & 16.816 & 2.253 & $0.50 \%$ & 16.123 & 2.678 \\
\hline $0.60 \%$ & 16.493 & 1.719 & $0.60 \%$ & 15.652 & 1.942 \\
\hline $0.70 \%$ & 16.365 & 2.822 & $0.70 \%$ & 15.178 & 2.642 \\
\hline $0.80 \%$ & 16.114 & 1.529 & $0.80 \%$ & 14.625 & 2.974 \\
\hline $0.90 \%$ & 16.245 & 2.223 & $0.90 \%$ & 14.325 & 2.912 \\
\hline $1.00 \%$ & 14.264 & 1.658 & $1.00 \%$ & 14.255 & 2.567 \\
\hline $2.00 \%$ & 13.507 & 1.658 & $2.00 \%$ & 14.162 & 2.871 \\
\hline $5.00 \%$ & 11.875 & 2.404 & $5.00 \%$ & 14.075 & 2.983 \\
\hline
\end{tabular}


Tabla 17. Tensiones Interfaciales - Pindo 13

\begin{tabular}{|c|c|c|c|c|c|}
\hline \multicolumn{3}{|c|}{ HALMX152805-1 } & \multicolumn{3}{|c|}{ HALMX152805-2 } \\
\hline Conc $(\%)$ & $\begin{array}{c}\text { IFT } \\
(\mathbf{m N} / \mathbf{m})\end{array}$ & $\begin{array}{l}\mathbf{E} \\
+-\end{array}$ & $\begin{array}{c}\text { Conc } \\
(\%)\end{array}$ & $\begin{array}{c}\text { IFT } \\
(\mathbf{m N} / \mathbf{m})\end{array}$ & $\begin{array}{l}\mathbf{E} \\
+-\end{array}$ \\
\hline $0 \%$ & 24.6515 & 2.786 & $0 \%$ & 22.935 & 4.585 \\
\hline $0.01 \%$ & 21.244 & 1.987 & $0.01 \%$ & 22.932 & 1.709 \\
\hline $0.05 \%$ & 20.6815 & 3.543 & $0.05 \%$ & 18.551 & 2.173 \\
\hline $0.10 \%$ & 18.335 & 1.654 & $0.10 \%$ & 18.176 & 2.009 \\
\hline $0.20 \%$ & 17.714 & 3.56 & $0.20 \%$ & 18.034 & 1.826 \\
\hline $0.30 \%$ & 16.531 & 3.789 & $0.30 \%$ & 17.022 & 1.837 \\
\hline $0.40 \%$ & 16.403 & 1.567 & $0.40 \%$ & 16.956 & 1.868 \\
\hline $0.50 \%$ & 16.304 & 2.678 & $0.50 \%$ & 16.756 & 2.02 \\
\hline $0.60 \%$ & 16.879 & 1.942 & $0.60 \%$ & 16.565 & 2.378 \\
\hline $0.70 \%$ & 16.301 & 3.785 & $0.70 \%$ & 16.375 & 2.809 \\
\hline $0.80 \%$ & 16.159 & 2.446 & $0.80 \%$ & 16.057 & 1.987 \\
\hline $0.90 \%$ & 16.109 & 3.168 & $0.90 \%$ & 16.001 & 1.876 \\
\hline $1.00 \%$ & 16.013 & 0.949 & $1.00 \%$ & 14.781 & 1.675 \\
\hline $2.00 \%$ & 14.274 & 3.112 & $2.00 \%$ & 13.089 & 2.987 \\
\hline $5.00 \%$ & 14.412 & 1.001 & $5.00 \%$ & 11.874 & 1.876 \\
\hline
\end{tabular}

Tabla 18. Tensiones Interfaciales - Pindo 17

\begin{tabular}{|c|c|c|c|c|c|}
\hline \multicolumn{3}{|c|}{ HALMX152805-1 } & \multicolumn{3}{|c|}{ HALMX152805-2 } \\
\hline Conc $(\%)$ & $\begin{array}{c}\text { IFT } \\
(\mathrm{mN} / \mathrm{m})\end{array}$ & $\begin{array}{l}E \\
+-\end{array}$ & $\begin{array}{c}\text { Conc } \\
(\%)\end{array}$ & $\begin{array}{c}\text { IFT } \\
(\mathbf{m N} / \mathbf{m})\end{array}$ & $\begin{array}{l}\mathbf{E} \\
+-\end{array}$ \\
\hline $0 \%$ & 34.6 & 5.468 & $0 \%$ & 37.502 & 1.716 \\
\hline $0.01 \%$ & 24.041 & 5.780 & $0.01 \%$ & 36.674 & 1.225 \\
\hline $0.05 \%$ & 23.204 & 6.277 & $0.05 \%$ & 33.752 & 0.984 \\
\hline $0.10 \%$ & 23.539 & 5.461 & $0.10 \%$ & 28.638 & 1.218 \\
\hline $0.20 \%$ & 23.462 & 2.829 & $0.20 \%$ & 23.638 & 2.69 \\
\hline $0.30 \%$ & 23.389 & 2.872 & $0.30 \%$ & 21.668 & 2.796 \\
\hline $0.40 \%$ & 23.283 & 5.156 & $0.40 \%$ & 20.302 & 3.546 \\
\hline $0.50 \%$ & 23.907 & 3 & $0.50 \%$ & 20.296 & 2.32 \\
\hline $0.60 \%$ & 23.793 & 5.964 & $0.60 \%$ & 20.243 & 2.52 \\
\hline $0.70 \%$ & 21.51 & 2.798 & $0.70 \%$ & 20.118 & 2.32 \\
\hline $0.80 \%$ & 21.189 & 2.645 & $0.80 \%$ & 20.261 & 3.332 \\
\hline $0.90 \%$ & 19.983 & 2.573 & $0.90 \%$ & 19.767 & 3.516 \\
\hline $1.00 \%$ & 19.345 & 2.48 & $1.00 \%$ & 19.999 & 1.21 \\
\hline $2.00 \%$ & 18.908 & 2.783 & $2.00 \%$ & 20.4 & 2.962 \\
\hline $5.00 \%$ & 18.898 & 3.821 & $5.00 \%$ & 20.345 & 3.062 \\
\hline
\end{tabular}

Tabla 19. Tensiones Interfaciales - Pindo 17

\begin{tabular}{|c|c|c|c|c|c|}
\hline \multicolumn{3}{|c|}{ BH - WCW2827 } & \multicolumn{3}{|c|}{ BH - WAW3037 } \\
\hline Conc (\%) & $\begin{array}{c}\text { IFT } \\
(\mathrm{mN} / \mathrm{m})\end{array}$ & $\begin{array}{l}\mathrm{E} \\
+-\end{array}$ & $\begin{array}{c}\text { Conc } \\
(\%)\end{array}$ & $\begin{array}{c}\text { IFT } \\
(\mathbf{m N} / \mathbf{m})\end{array}$ & $\begin{array}{l}E \\
+-\end{array}$ \\
\hline $0 \%$ & 32.604 & 6.895 & $0 \%$ & 34.6 & 5.468 \\
\hline $0.01 \%$ & 26.132 & 4.613 & $0.01 \%$ & 24.041 & 5.780 \\
\hline $0.05 \%$ & 25.132 & 2.949 & $0.05 \%$ & 23.204 & 6.277 \\
\hline $0.10 \%$ & 24.738 & 3.044 & $0.10 \%$ & 23.539 & 5.461 \\
\hline $0.20 \%$ & 24.499 & 5.335 & $0.20 \%$ & 23.462 & 2.829 \\
\hline $0.30 \%$ & 23.647 & 2.955 & $0.30 \%$ & 23.389 & 2.872 \\
\hline $0.40 \%$ & 24.909 & 3.147 & $0.40 \%$ & 23.283 & 5.156 \\
\hline $0.50 \%$ & 24.824 & 5.711 & $0.50 \%$ & 23.907 & 3 \\
\hline $0.60 \%$ & 23.45 & 11.071 & $0.60 \%$ & 23.793 & 5.964 \\
\hline $0.70 \%$ & 24.349 & 3.129 & $0.70 \%$ & 21.51 & 2.798 \\
\hline $0.80 \%$ & 24.561 & 1.175 & $0.80 \%$ & 21.189 & 2.645 \\
\hline $0.90 \%$ & 24.349 & 3.129 & $0.90 \%$ & 19.983 & 2.573 \\
\hline $1.00 \%$ & 22.887 & 4.705 & $1.00 \%$ & 19.345 & 2.48 \\
\hline $2.00 \%$ & 21.732 & 2.539 & $2.00 \%$ & 18.908 & 2.783 \\
\hline $5.00 \%$ & 21.529 & 2.539 & $5.00 \%$ & 18.898 & 3.821 \\
\hline
\end{tabular}

\section{ABREVIATURAS}

IFT: Interfacial tension o Tensión interfacial.

TS: Tensión Superficial.

IR: Infrared Spectroscopy o espectro infrarrojo.

BSW: Basic Sediment Water.

$\mathbf{m N}$ : miliNewtons

m: metro

$\%$ : Porcentaje

ms: miliSiemens.

mg/l: Miligramo por cada litro

ppm: Partes por millón

TDS: Total de sólidos disueltos

MSCFPD: Millones de pies cúbicos por día

BOPD: Barriles de petróleo por día

BWPD: Barriles de agua por día

\%BSW: Porcentaje Basic Sediment Water

CMC: Concentración micelar crítica

Ui: Arena productora U inferior

${ }^{\circ} \mathrm{C}$ : Grados Celsius

API: American Petroleum Institute

\% T: Porcentaje de Transmitancia.

Conc: Concentración

E: Error

Cond: Conductividad 
Revista Politécnica, Mayo - Julio 2021, Vol. 47, No. 2 